\title{
Inovação e colaboração no turismo carioca: Primeiras evidências empíricas
}

\section{Innovation and cooperation in carioca's tourism: First empirical evidence}

\author{
Innovación y cooperación en el turismo carioca: \\ Las primeras evidencias empíricas
}

\author{
Mariana de Queiroz Brunelli ${ }^{1}$ \\ T. Diana L. v. A. de Macedo-Soares ${ }^{2}$ \\ Roberto Farias Silva ${ }^{3}$
}

\begin{abstract}
Resumo: Este artigo apresenta os resultados de uma pesquisa que analisou a relação entre colaboração e inovação na indústria do turismo, a fim de verificar se os relacionamentos colaborativos entre firmas contribuem ao desenvolvimento de inovações. As informações foram coletadas por meio de uma survey realizada com representantes de organizações atuantes no turismo da cidade do Rio de Janeiro, destino particularmente interessante por ter sido escolhido como sede de grandes eventos, precisamente a Copa do Mundo da FIFA, em 2014, e os Jogos Olímpicos, em 2016. A análise e a interpretação dos dados foram realizadas por meio de técnicas estatísticas. Os resultados evidenciaram que os relacionamentos colaborativos entre as organizações pesquisadas contribuíram de forma significativa para o desenvolvimento de inovações no turismo do destino pesquisado. A análise dos resultados evidenciou ainda o potencial latente de transformação do turismo carioca por meio de inovações ao se fortalecerem as parcerias identificadas nesta pesquisa no sentido de gerarem produtos com maior valor agregado e, ao mesmo tempo, com práticas de precificação coerentes a nível global.
\end{abstract}

Palavras-chave: Inovação; Colaboração; Turismo; Rio de Janeiro; Brasil.

Abstract: This article presents the results of a study that examined the relationship between collaboration and innovation in the tourism industry in order to verify if the collaborative relationships between firms contribute to the development of innovations. Information was collected through a survey conducted with representatives of organizations active in tourism in the city of Rio de Janeiro, a particularly interesting destination due to it is having been chosen to host major events, specifically the FIFA World Cup in 2014, and the Olympic Games in 2016. Analysis and interpretation of data were performed by using statistical techniques. The results showed that the collaborative relationships between the organizations surveyed have contributed significantly to the development of innovations in the tourism destination focused. The results also made evident the latent potential for transformation of tourism in Rio de Janeiro through innovations, by strengthening the partnerships identified in this research, in order to generate products with

\footnotetext{
${ }^{1}$ Pesquisadora do Departamento de Administração da PUC-Rio. E-mail: maribrunelli@gmail.com

${ }^{2}$ Professora Titular do Departamento de Administração da PUC-Rio. E-mail:

tdiana.vanaduardmacedosoares@gmail.com

3 Professor do Departamento de Turismo do Senac-Rio.E-mail: roberto.farias@gmail.com
} 
higher added value and, at the same time, with globally consistent pricing practices.

Keywords: Innovation; Collaboration; Tourism; Rio de Janeiro; Brazil.

Resumen: Este artículo presenta los resultados de un estudio que examinó la relación entre la colaboración y la innovación en la industria del turismo con el fin de verificar que las relaciones de colaboración entre las empresas poden contribuir al desarrollo de innovaciones. Se recolectó información a través de una encuesta llevada a cabo con los representantes de las organizaciones que trabajan en el turismo en la ciudad de Río de Janeiro, el destino es particularmente interesante por haber sido elegida para acoger grandes eventos, en particular la Copa Mundial de la FIFA en 2014, y los Juegos Olímpicos de 2016. El análisis e interpretación de los datos se realizaron mediante el uso de técnicas estadísticas. Los resultados mostraron que las relaciones de colaboración entre las organizaciones encuestadas han contribuido significativamente al desarrollo de innovaciones en el destino turístico. Los resultados también mostraron el potencial latente para la transformación del turismo en Río de Janeiro a través de innovaciones para reforzar las asociaciones identificadas en esta investigación con el fin de generar productos con mayor valor agregado y, al mismo tiempo, consistentes a nivel mundial.

Palabras clave: Innovación; Colaboración; Turismo; Río de Janeiro; Brasil.

\section{INTRODUÇÃO}

A indústria do turismo tem passado por notório processo de globalização (Hjalager, 2007). O aumento da competitividade e as rápidas transformações vividas pelas organizações turísticas têm gerado pressões para que elas inovem e para que estabeleçam novas configurações organizacionais, tais como alianças e redes. Nesse contexto, o tema da inovação tem adquirido crescente relevância na literatura sobre turismo nas últimas duas décadas (Sundbo, Orfila-Sintes \& Sørensen, 2007; Hjalager, 2010).

Embora o turismo ainda seja considerado uma indústria moderadamente inovadora, questões relativas à inovação têm ganhado cada vez mais espaço na criação de políticas para o turismo e no desenvolvimento de produtos, empresas e destinos mais competitivos pelo mundo (Hall, 2009). Evidências apontam para o fato de que a inovação no turismo pode gerar vantagens competitivas, lealdade de consumidores e redução de custos, que são fundamentais para o crescimento de um destino (Victorino, Verma, Plaschka \& Dev, 2005; Buhalis, 1999).

De acordo com Hjalager (2010), entretanto, muitas questões ainda estão em aberto no campo da inovação em turismo. Com base na sua revisão do estado da arte sobre esse tema, essa autora verificou que ainda há poucos trabalhos sistematizados e comparáveis sobre o nível de atividades inovadoras das organizações que atuam no turismo e seus impactos e implicações mais amplas para os destinos e para as economias nacionais. Ficou evidente que novos estudos quantitativos e qualitativos ainda são necessários para consolidar a pesquisa sobre o tema (Hjalager, 2010).

Nesse sentido, esse artigo apresenta os primeiros resultados de uma pesquisa que buscou verificar se os relacionamentos colaborativos entre firmas da indústria do turismo são importantes para o desenvolvimento de inovações que possam contribuir para a vantagem competitiva das empresas e do destino. A relevância da pesquisa está em contribuir não só para a construção da 
teoria sobre inovação em turismo, por meio da colaboração, mas também para a obtenção de um maior entendimento a respeito do processo de inovação das empresas envolvidas no turismo do destino pesquisado. Precisamente, no caso deste artigo, enfoca-se a cidade do Rio de Janeiro, no Brasil. Este destino é particularmente relevante porquanto será foco de grandes investimentos nos próximos anos em função de sediar megaeventos mundiais, principalmente a Copa do Mundo da FIFA, em 2014, e os Jogos Olímpicos, em 2016. Além disso, ainda não há literatura acadêmica publicada sobre inovação no turismo que investigue este destino em particular quanto às questões aqui abordadas.

Este artigo foi organizado em seis seções. Após esta introdução, o referencial teórico foi descrito. Em seguida, a metodologia da pesquisa foi explicitada e, na seção seguinte, os resultados foram apresentados. Na quinta seção, os principais achados da pesquisa foram discutidos e, na sequência, as considerações finais foram formuladas.

\section{TURISMO E INOVAÇÃO}

O arcabouço teórico Schumpeteriano (1934), que versa sobre a principal fonte de crescimento econômico ser gerada por novas formas de combinação dos meios de produção, é a principal base da literatura sobre inovação no turismo (Hjalager, 2002; Matínez-Ros \& OrfilaSintes, 2009; Mayer, 2009). Segundo Hjalager (2002, 2010), tal conceito amplo de inovação é adequado à indústria do turismo e deve contemplar adaptações menores em produtos e serviços existentes, ações que se diferenciam da forma usual de fazer negócios ou que representam descontinuidades de práticas adotadas. Nesse sentido, mesmo sendo considerada uma indústria moderadamente inovadora, o ato de inovar é apontado por pesquisadores como uma condição de sobrevivência para as empresas que operam no ambiente extremamente competitivo e em contínua transformação do turismo (Sundbo et al., 2007).

Apesar de ainda não ter sido desenvolvida uma compreensão abrangente sobre as forças que movem a inovação no turismo (Hjalager, 2010), algumas descobertas têm sido apresentadas. Sundbo et al. (2007) demonstram que tamanho, profissionalismo, empreendedorismo e uma rede variada de relacionamentos são importantes determinantes de inovação no turismo. A questão do tamanho tanto das empresas quanto dos destinos para a inovação é reforçada pelos trabalhos de Orfila-Sintes, Crespi-Cladera e Martinez-Ros (2005), Pikkemaat e Peters (2005) e Matínez-Ros e Orfila-Sintes (2009).

Já a necessidade de estabelecer relacionamentos de colaboração é apontada também por outros autores como fundamental para a transferência de conhecimentos e recursos necessários à inovação no turismo (Pavlovich, 2003; Pikkemaat \& Weiermair, 2007; Pikkemaat, 2008). Tamanho e colaboração como indicadores de inovação tiveram respaldo no trabalho de Rodgers (2004) quando analisa empresas de serviços. Hjalager (2010) acrescenta que o investimento em tecnologias de informação e comunicação, que podem gerar rápidos impactos nas práticas mercadológicas, é outro fator de inovação importante no turismo. No entanto, essa autora 
considera a demanda de mercado o principal motor para que as organizações turísticas possam inovar (Hjalager, 2010).

Entre os tipos de inovação que são encontrados no turismo, a classificação de Hjalager $(1997,2010)$ em cinco categorias pode ser considerada adequada para fins analíticos. A "inovação em produto ou serviço" consiste no desenvolvimento de novos produtos ou serviços ou no aprimoramento daqueles já existentes visando acrescentar valor para os clientes e expandir a variedade de experiências ofertadas. A "inovação em processo", por sua vez, diz respeito ao desenvolvimento de tecnologias ou novas formas de organização que facilitam a execução das atividades do negócio. No caso da "inovação em administração", o foco recai sobre o desenvolvimento ou atualização da forma como o negócio, a empresa, a rede de parceiros e os colaboradores são organizados. Já a categoria "inovação em gestão" diz respeito ao desenvolvimento de novos processos ou tecnologias para gestão, por exemplo, de materiais, transações, informações e fornecimento como no uso de Internet marketing, no aprimoramento dos sistemas de aeroportos e outros sistemas de informações integrados sobre o destino. A última categoria nessa classificação é a "inovação institucional" que engloba o desenvolvimento de novas estruturas de colaboração e regulação no destino, que redirecionem ou aumentem os negócios em certos campos do turismo.

Alguns autores (Mayer, 2009; Hjalager, 2002), no entanto, consideram que outros setores ligados ao turismo podem ter mais responsabilidade na introdução de inovações nessa indústria, como é caso das indústrias de transportes e comunicações. Esse talvez seja o motivo de poucas políticas públicas reconhecerem o turismo como uma indústria que pode contribuir para a inovação e a competitividade de uma cidade ou país (Hall, 2009).

\section{INOVAÇÃO E COLABORAÇÃO NO TURISMO}

Scott, Cooper e Baggio (2008) afirmam que o turismo, mais do que outros setores econômicos, depende do desenvolvimento formal e informal de relações de colaboração, parcerias e redes. Wang e Krakover (2009) justificam essa necessidade pela fragmentação e complexidade dessa indústria. Realmente, as empresas que atuam no turismo estão cada vez mais gerenciando as incertezas do ambiente por meio do estabelecimento de redes de ligações que englobam trocas de informação, construção de canais de comunicação confiáveis e planejamento conjunto (Tremblay, 1998).

Não é por acaso que a colaboração é vista como um fator importante para o desenvolvimento de inovações na indústria do turismo (Pikkemaat \& Weiermair, 2007; Sundbo et al., 2007; Pavlovich, 2003). Conforme afirma Hjalager (2010), a literatura sobre turismo deixa claro que os relacionamentos inter-firmas são cruciais para a transferência de informações, inspirações e inovações. Para Stamboulis e Skayannis (2003), são os relacionamentos baseados em confiança que permitem os processos de inovação evoluir até que os investimentos se sustentem.

De fato, as evidências encontradas na literatura mostram que os relacionamentos de 
colaboração são positivamente correlacionados com o nível de inovação das empresas turísticas (Pikkemaat \& Weiermair, 2007). Segundo Sundbo et al. (2007), isso acontece devido as empresas precisarem nos seus processos de inovação de parceiros externos e de trajetórias de conhecimento para complementar suas competências. Pavlovich (2003) argumenta que a rede de relacionamentos das empresas é um fator crítico para o desenvolvimento de um destino turístico, pois ela contribui para trocas intensivas de informação que favorecem a geração de conhecimento compartilhado entre os parceiros. Esses fatos, conforme colocam Pikkemaat e Weiermair (2007), enfatizam a importância de fortalecer as estratégias de colaboração para que as empresas que atuam no turismo possam atingir um nível maior de inovação.

Entretanto, apesar dos relacionamentos de colaboração serem apontados como importantes para o desenvolvimento de inovações, alguns estudos indicam que eles ainda são limitados na indústria do turismo (Hjalager, 2010; Plaza, Galvez-Galvez \& Gonzalez-Flores, 2011). Por exemplo, os relacionamentos de colaboração com universidades, mencionados como fundamentais para o desenvolvimento de inovações na indústria de manufaturados, não são destacados com a mesma relevância na indústria do turismo (Hjalager, 2010). Por outro lado, Hjalager (2010) afirma que há uma grande ênfase nos relacionamentos colaborativos para o desenvolvimento de políticas para o turismo e que os governos são poderosos parceiros na coprodução de instalações turísticas nos destinos.

\section{PRINCIPAIS ATORES DA INDÚSTRIA DO TURISMO}

Para a pesquisa, cujos resultados são apresentados neste artigo, era fundamental conhecer os principais atores da indústria do turismo de modo a analisar a relação entre colaboração e desenvolvimento de inovação. Pela literatura, é possível verificar que os principais atores da rede do turismo são aqueles identificados como os que possuem uma posição mais central entre as empresas e organizações turísticas (Timur \& Getz, 2008). De acordo com Timur e Getz (2008), um alto nível de centralidade significa que esses atores são mais capazes de gerenciar fluxos de informação e influenciar outros atores, caracterizando uma vantagem estratégica para eles.

Além disso, à luz dos conceitos da teoria de stakeholders, Timur e Getz (2008) mostram que a identificação dos atores críticos ao desenvolvimento do turismo está relacionada à análise de dois atributos fundamentais: o poder e a legitimidade exercidos por eles em relação aos outros atores da rede do turismo.

Nessa linha, segundo Scott et al. (2008), os principais atores da indústria do turismo são aqueles com interesse mais amplo em relação às questões de um destino. Eles seriam empresas maiores com rendimentos provenientes de vários setores da indústria e envolvidos em mais de uma comunidade de interesse no destino, tal como grandes hotéis.

Entre os principais atores da rede do turismo, incluem-se também as organizações responsáveis pelo gerenciamento das ações de promoção do destino como os "Convention \& Visitors Bureaux", as agências de viagens, as operadoras turísticas e as entidades governamentais 
e de classe (Timur \& Getz, 2008; Donaire, Silva \& Gaspar, 2009; March \& Wilkinson, 2009).

A importância desses atores para a indústria do turismo se dá pelas responsabilidades atribuídas a esses agentes. Dentre essas, destacam-se a comunicação do planejamento de desenvolvimento do destino, a mediação para facilitar a colaboração entre os stakeholders, a crescente necessidade de sensibilizar os membros da rede em relação às questões socioambientais e à coordenação dos esforços para o alcance das metas coletivas da indústria do turismo (Timur \& Getz, 2008).

\section{METODOLOGIA}

A pesquisa em questão neste artigo foi essencialmente exploratória e descritiva (Gil, 2009; Stevenson, 2002). Em relação ao método de coleta de dados, tratou-se de uma pesquisa quantitativa do tipo survey, realizada a partir de uma amostra de gestores atuantes nas empresas do setor de turismo. A amostra considerada intencional foi montada com o auxilio de especialistas do setor, especialmente para identificar as maiores empresas envolvidas, uma vez que essa variável é determinante de inovação de acordo com a literatura. A unidade de observação foi formada por profissionais em nível de gerência ou direção. Obteve-se uma amostra final de 92 organizações respondentes, número aproximado ao tamanho calculado para a amostra mínima, em torno de 90 respondentes, atendendo às exigências de amostragem estatística, assim como, aos objetivos estabelecidos para o estudo. Foi constituída por 37 hotéis, 28 agências de viagem, 13 operadoras de turismo, 6 agências promotoras do turismo, 7 associações representativas das empresas do turismo e 1 organização governamental voltada para o turismo.

$\mathrm{O}$ instrumento de coleta de dados utilizado na pesquisa foi um questionário formado por questões mensuradas por meio de escalas do tipo Likert (1975), variando de "Discordo Completamente" a "Concordo Completamente", perfazendo um total de cinco pontos. Os questionários foram preenchidos, no período de novembro 2011 a fevereiro 2012, em entrevistas presenciais, o que possibilitou o esclarecimento de qualquer dúvida do entrevistado.

Os dados coletados por intermédio dos questionários impressos foram inicialmente tabulados no software de elaboração de planilhas eletrônicas da Microsoft - Excel - , com o objetivo de serem, posteriormente, processados por meio do software estatístico Statistical Package for the Social Sciences (SPSS), da IBM. Efetuada a tabulação inicial dos dados, passou-se à realização de uma análise exploratória, na qual se constatou dados ausentes, que levaram a amostra a ser reduzida de 97 para 92 questionários válidos.

A etapa seguinte ao processo de tratamento estatístico dos dados consistiu na aplicação das técnicas estatísticas de análise da consistência interna dos questionários, por meio do Alfa de Cronbach, e do teste de correlação de Sperman, por se tratar de variáveis qualitativas ordinais, visando estudar a possível existência de correlação entre relacionamentos colaborativos e o desenvolvimento de inovações para os seguintes atores atuantes na indústria do turismo da cidade do Rio de Janeiro: hotéis, agências de viagem, operadoras de turismo, organizações 
promotoras do destino, instituições de ensino e pesquisa em turismo, entidades de classe e organizações governamentais.

Ressalta-se que todos os desenvolvimentos estatísticos descritivos e inferenciais utilizados neste artigo estão devidamente fundamentados e comprovados por demonstrações específicas na bibliografia referenciada (Stevenson, 2002; Hair, Anderson, Tatham \& Black, 2006) e, por esta razão, deixou-se de demonstrar aqui os teoremas pertinentes.

\section{RESULTADOS}

Coerente com o objetivo da pesquisa, os resultados aqui apresentados visaram verificar se, na percepção dos executivos entrevistados, os relacionamentos colaborativos com outras firmas que atuam no turismo contribuem ao desenvolvimento de inovações. Para a análise dos dados, foram utilizados métodos quantitativos baseados em estatística descritiva (percentagens simples e cálculo da correlação de Sperman) e em inferência estatística (teste não paramétrico de correlação). Ademais, primeiramente, foi analisada a consistência interna das informações nos questionários via o cálculo do Alfa de Cronbach, obtendo-se uma alta confiabilidade de 86,4\%.

Em relação às estatísticas descritivas, pode-se dizer que a grande maioria dos gestores pesquisados concordou que as relações da sua organização com outros atores do turismo eram muito importantes para o desenvolvimento de inovações, conforme dados destacados na Tabela 1.

Tabela 1. Importância dos relacionamentos para o desenvolvimento de inovações

\begin{tabular}{|c|c|c|c|c|c|c|c|}
\hline $\begin{array}{l}\text { "Os relacionamentos da minha empresa com as } \\
\text { organizações abaixo são muito importantes para o } \\
\text { desenvolvimento de inovações e/ou vantagens } \\
\text { competitivas no meu negócio". }\end{array}$ & Hotéis & $\begin{array}{l}\text { Agência de } \\
\text { viagens }\end{array}$ & $\begin{array}{l}\text { Operadoras } \\
\text { de turismo }\end{array}$ & $\begin{array}{l}\text { Inst. de } \\
\text { Ensino e } \\
\text { Pesquisa }\end{array}$ & $\begin{array}{c}\text { Associações } \\
\text { de classe }\end{array}$ & $\begin{array}{c}\text { Org. } \\
\text { Promotoras } \\
\text { do destino }\end{array}$ & $\begin{array}{c}\text { Org. } \\
\text { Governo }\end{array}$ \\
\hline Discordo completamente & $0,00 \%$ & $1,10 \%$ & $2,20 \%$ & $1,10 \%$ & $1,10 \%$ & $1,10 \%$ & $1,10 \%$ \\
\hline Discordo & $1,10 \%$ & $2,20 \%$ & $3,30 \%$ & $2,20 \%$ & $3,30 \%$ & $2,20 \%$ & $5,40 \%$ \\
\hline Nem concordo/nem discordo & $5,40 \%$ & $4,30 \%$ & $12,00 \%$ & $19,60 \%$ & $19,60 \%$ & $15,20 \%$ & $17,40 \%$ \\
\hline Concordo & $35,90 \%$ & $34,80 \%$ & $37,00 \%$ & $42,40 \%$ & $39,10 \%$ & $42,40 \%$ & $37,00 \%$ \\
\hline Concordo completamente & $53,30 \%$ & $52,20 \%$ & $43,50 \%$ & $32,60 \%$ & $33,70 \%$ & $38,00 \%$ & $38,00 \%$ \\
\hline$\%$ de respondentes totais por questão & $95,70 \%$ & $94,60 \%$ & $97,80 \%$ & $97,80 \%$ & $96,70 \%$ & $98,90 \%$ & $98,90 \%$ \\
\hline
\end{tabular}

Além disso, os relacionamentos investigados foram reportados, em maioria, como formais (Tabela 2) e colaborativos (Tabela 3).

Tabela 2. Formalização dos relacionamentos pesquisados

\begin{tabular}{|c|c|c|c|c|c|c|c|}
\hline $\begin{array}{l}\text { "A maioria dos relacionamentos da minha empresa } \\
\text { com as organizações abaixo são formais". }\end{array}$ & Hotéis & $\begin{array}{l}\text { Agência de } \\
\text { viagens }\end{array}$ & $\begin{array}{l}\text { Operadoras } \\
\text { de turismo }\end{array}$ & $\begin{array}{l}\text { Inst. de } \\
\text { Ensino e } \\
\text { Pesquisa }\end{array}$ & $\begin{array}{l}\text { Associações } \\
\text { de classe }\end{array}$ & $\begin{array}{c}\text { Org. } \\
\text { Promotoras } \\
\text { do destino }\end{array}$ & $\begin{array}{c}\text { Org. } \\
\text { Governo }\end{array}$ \\
\hline Discordo completamente & $4,30 \%$ & $2,20 \%$ & $2,20 \%$ & $7,60 \%$ & $3,30 \%$ & $3,30 \%$ & $2,20 \%$ \\
\hline Discordo & $13,00 \%$ & $6,50 \%$ & $10,90 \%$ & $13,00 \%$ & $8,70 \%$ & $13,00 \%$ & $15,20 \%$ \\
\hline Nem concordo/nem discordo & $7,60 \%$ & $8,70 \%$ & $7,60 \%$ & $21,70 \%$ & $16,30 \%$ & $18,50 \%$ & $17,40 \%$ \\
\hline Concordo & $28,30 \%$ & $29,30 \%$ & $31,50 \%$ & $28,30 \%$ & $43,50 \%$ & $31,50 \%$ & $25,00 \%$ \\
\hline$\%$ de respondentes totais por questão & $92,40 \%$ & $90,20 \%$ & $93,50 \%$ & $90,20 \%$ & $96,70 \%$ & $95,70 \%$ & $95,70 \%$ \\
\hline
\end{tabular}


Brunelli, M. de Q., Macedo-Soares, T. D. L. v. A., \& Silva, R. F. Inovação e colaboração no turismo carioca: Primeiras evidências empíricas

Tabela 3. Colaboração nos relacionamentos pesquisados

\begin{tabular}{|c|c|c|c|c|c|c|c|}
\hline $\begin{array}{l}\text { "Na maioria dos relacionamentos da minha empresa } \\
\text { com as organizações abaixo não há vantagens } \\
\text { desproporcionais entre os parceiros e o resultado da } \\
\text { parceria gera benefícios para ambas as partes". }\end{array}$ & Hotéis & $\begin{array}{l}\text { Agência de } \\
\text { viagens }\end{array}$ & $\begin{array}{c}\text { Operadoras } \\
\text { de turismo }\end{array}$ & $\begin{array}{l}\text { Inst. de } \\
\text { Ensino e } \\
\text { Pesquisa }\end{array}$ & $\begin{array}{c}\text { Associações } \\
\text { de classe }\end{array}$ & $\begin{array}{c}\text { Org. } \\
\text { Promotoras } \\
\text { do destino }\end{array}$ & $\begin{array}{c}\text { Org. } \\
\text { Governo }\end{array}$ \\
\hline Discordo completamente & $1,10 \%$ & $3,30 \%$ & $2,20 \%$ & $2,20 \%$ & $3,30 \%$ & $3,30 \%$ & $5,40 \%$ \\
\hline Discordo & $5,40 \%$ & $4,30 \%$ & $2,20 \%$ & $2,20 \%$ & $3,30 \%$ & $3,30 \%$ & $4,30 \%$ \\
\hline Nem concordo/nem discordo & $12,00 \%$ & $10,90 \%$ & $15,20 \%$ & $27,20 \%$ & $28,30 \%$ & $25,00 \%$ & $26,10 \%$ \\
\hline Concordo & $39,10 \%$ & $26,10 \%$ & $37,00 \%$ & $39,10 \%$ & $35,90 \%$ & $29,30 \%$ & $33,70 \%$ \\
\hline Concordo completamente & $39,10 \%$ & $43,50 \%$ & $38,00 \%$ & $22,80 \%$ & $19,60 \%$ & $30,40 \%$ & $22,80 \%$ \\
\hline$\%$ de respondentes totais por questão & $96,70 \%$ & $88,00 \%$ & $94,60 \%$ & $93,50 \%$ & $90,20 \%$ & $91,30 \%$ & $92,40 \%$ \\
\hline
\end{tabular}

Por outro lado, a periodicidade com que os parceiros afirmaram se relacionar foi diferente para cada categoria de ator. Os atores com os quais os gestores reportaram se relacionar com maior frequência (mensal ou quinzenal) foram os hotéis (74\%), as agências de viagem (72\%), as operadoras de turismo (63\%) e as organizações promotoras do destino (41\%). Já os relacionamentos com institutos de ensino e pesquisa (47\%), associações de classe (40\%), organizações governamentais (50\%) foram relatados como os de contato menos frequente.

Em relação aos tipos de recursos mais compartilhados nos relacionamentos pesquisados, capital informacional foi um destaque em todas as relações reportadas, conforme mostra a Tabela 4. O capital social foi um recurso bastante citado também nos relacionamentos com agências de viagens (18,5\%), operadoras de turismo $(15,2 \%)$, institutos de ensino e pesquisa $(22,8 \%)$ e organizações promotoras do destino (10,9\%). No caso dos relacionamentos com hotéis destacou-se ainda o compartilhamento de recursos físicos (21,7\%) e nas relações com as organizações governamentais, os recursos financeiros $(19,6 \%)$.

Tabela 4. Recursos compartilhados nos relacionamentos pesquisados

\begin{tabular}{|c|c|c|c|c|c|c|c|}
\hline $\begin{array}{c}\text { "Considerando o conjunto dos relacionamentos da sua } \\
\text { empresa com as organizações que compõe as } \\
\text { categorias abaixo, indique que tipos de recursos são } \\
\text { mais acessados pela sua empresa nesses } \\
\text { relacionamentos". }\end{array}$ & Hotéis & $\begin{array}{l}\text { Agência de } \\
\text { viagens }\end{array}$ & $\begin{array}{l}\text { Operadoras } \\
\text { de turismo }\end{array}$ & $\begin{array}{l}\text { Inst. de } \\
\text { Ensino e } \\
\text { Pesquisa }\end{array}$ & $\begin{array}{c}\text { Associações } \\
\text { de classe }\end{array}$ & $\begin{array}{c}\text { Org. } \\
\text { Promotoras } \\
\text { do destino }\end{array}$ & $\begin{array}{c}\text { Org. } \\
\text { Governo }\end{array}$ \\
\hline Capital social & $13,00 \%$ & $18,50 \%$ & $15,20 \%$ & $22,80 \%$ & $15,20 \%$ & $10,90 \%$ & $12,00 \%$ \\
\hline Capital informacional & $26,10 \%$ & $15,20 \%$ & $21,70 \%$ & $19,60 \%$ & $37,00 \%$ & $30,40 \%$ & $28,30 \%$ \\
\hline Recursos financeiros & $2,20 \%$ & $7,60 \%$ & $9,80 \%$ & $5,40 \%$ & $3,30 \%$ & $9,80 \%$ & $19,60 \%$ \\
\hline Recursos tecnológicos & $4,30 \%$ & $1,10 \%$ & $1,10 \%$ & $6,50 \%$ & $2,20 \%$ & $5,40 \%$ & $5,40 \%$ \\
\hline$\%$ de respondentes totais por questão & $67,40 \%$ & $53,30 \%$ & $58,70 \%$ & $62,00 \%$ & $59,80 \%$ & $58,70 \%$ & $68,50 \%$ \\
\hline
\end{tabular}

Ademais, a maioria dos gestores concordou que os relacionamentos da sua organização com os outros atores que também atuam no turismo proporcionam o desenvolvimento de inovações, conforme Tabela 5. 
Tabela 5. Desenvolvimento de inovação por meio dos relacionamentos pesquisados

\begin{tabular}{|c|c|c|c|c|c|c|c|}
\hline $\begin{array}{l}\text { "A maioria dos relacionamentos da minha empresa } \\
\text { com as organizações abaixo proporciona o } \\
\text { desenvolvimento de inovações no meu negócio". }\end{array}$ & Hotéis & $\begin{array}{l}\text { Agência de } \\
\text { viagens }\end{array}$ & $\begin{array}{l}\text { Operadoras } \\
\text { de turismo }\end{array}$ & $\begin{array}{l}\text { Inst. de } \\
\text { Ensino e } \\
\text { Pesquisa }\end{array}$ & $\begin{array}{c}\text { Associações } \\
\text { de classe }\end{array}$ & $\begin{array}{c}\text { Org. } \\
\text { Promotoras } \\
\text { do destino }\end{array}$ & $\begin{array}{c}\text { Org. } \\
\text { Governo }\end{array}$ \\
\hline Discordo completamente & $3,30 \%$ & $2,20 \%$ & $2,20 \%$ & $7,60 \%$ & $4,30 \%$ & $4,30 \%$ & $7,60 \%$ \\
\hline Discordo & $7,60 \%$ & $9,80 \%$ & $10,90 \%$ & $5,40 \%$ & $7,60 \%$ & $6,50 \%$ & $5,40 \%$ \\
\hline Nem concordo/nem discordo & $19,60 \%$ & $17,40 \%$ & $22,80 \%$ & $26,10 \%$ & $32,60 \%$ & $25,00 \%$ & $31,50 \%$ \\
\hline Concordo & $40,20 \%$ & $32,60 \%$ & $31,50 \%$ & $40,20 \%$ & $38,00 \%$ & $39,10 \%$ & $34,80 \%$ \\
\hline Concordo completamente & $25,00 \%$ & $33,70 \%$ & $23,90 \%$ & $13,00 \%$ & $10,90 \%$ & $18,50 \%$ & $13,00 \%$ \\
\hline$\%$ de respondentes totais por questão & $95,70 \%$ & $95,70 \%$ & $91,30 \%$ & $92,40 \%$ & $93,50 \%$ & $93,50 \%$ & $92,40 \%$ \\
\hline
\end{tabular}

Dentre os tipos de inovação desenvolvidos, por meio dos relacionamentos estabelecidos entre as organizações que atuam no turismo, os gestores informaram que as parcerias com hotéis $(14,1 \%)$, agências de viagem $(12 \%)$ e operadoras de turismo $(8,7 \%)$ geraram mais inovações em produtos e serviços. As instituições de ensino e pesquisa foram consideradas parceiras para 0 desenvolvimento de inovações em gestão e institucionais, assim como as associações de classe. Por sua vez, as organizações promotoras dos destinos foram reportadas como parceiros para o desenvolvimento de inovações institucionais e também logísticas. Já as organizações governamentais foram apontadas como parceiras principalmente no caso das inovações institucionais.

Tabela 6. Tipo de inovação desenvolvido por meio dos relacionamentos pesquisados

\begin{tabular}{|c|c|c|c|c|c|c|c|}
\hline $\begin{array}{c}\text { "Considerando o conjunto dos relacionamentos da sua } \\
\text { empresa com as organizações que compõe as } \\
\text { categorias abaixo, indique que tipos de inovação são } \\
\text { geralmente desenvolvidos por meio desses } \\
\text { relacionamentos". } \\
\end{array}$ & Hotéis & $\begin{array}{l}\text { Agência de } \\
\text { viagens }\end{array}$ & $\begin{array}{l}\text { Operadoras } \\
\text { de turismo }\end{array}$ & $\begin{array}{l}\text { Inst. de } \\
\text { Ensino e } \\
\text { Pesquisa }\end{array}$ & $\begin{array}{c}\text { Associações } \\
\text { de classe }\end{array}$ & \begin{tabular}{|c} 
Org. \\
Promotoras \\
do destino
\end{tabular} & $\begin{array}{c}\text { Org. } \\
\text { Governo }\end{array}$ \\
\hline Inovação em produto e serviço & $14,10 \%$ & $12,00 \%$ & $8,70 \%$ & $7,60 \%$ & $5,40 \%$ & $9,80 \%$ & $5,40 \%$ \\
\hline Inovação em processo & $6,50 \%$ & $6,50 \%$ & $5,40 \%$ & $3,30 \%$ & $2,20 \%$ & $3,30 \%$ & $6,50 \%$ \\
\hline Inovação em gestão & $1,10 \%$ & $3,30 \%$ & $4,30 \%$ & $10,90 \%$ & $8,70 \%$ & $4,30 \%$ & $4,30 \%$ \\
\hline Inovação em logística & $7,60 \%$ & $9,80 \%$ & $6,50 \%$ & $3,30 \%$ & $5,40 \%$ & $10,90 \%$ & $5,40 \%$ \\
\hline Inovações institucionais & $1,10 \%$ & $0,00 \%$ & $1,10 \%$ & $8,70 \%$ & $15,20 \%$ & $14,10 \%$ & $29,30 \%$ \\
\hline$\%$ de respondentes totais por questão & $30,40 \%$ & $31,50 \%$ & $26,10 \%$ & $33,70 \%$ & $37,00 \%$ & $42,40 \%$ & $51,10 \%$ \\
\hline
\end{tabular}

Para aprofundar a análise, o coeficiente de correlação de Spearman foi calculado utilizando-se como base as respostas para as questões 1 ("os relacionamentos da minha empresa com as organizações abaixo são muito importantes para o desenvolvimento de inovações e/ou vantagens competitivas?") e 5 ("A maioria dos relacionamentos da minha empresa com as organizações abaixo proporciona na prática o desenvolvimento de inovações no meu negócio"). Ou seja, buscou-se analisar a correlação entre a percepção de importância dos relacionamentos estabelecidos com a percepção da prática de inovações nesses mesmos relacionamentos.

Para isso, testes de hipóteses foram realizados para cada um dos atores com os quais os respondentes poderiam ter um relacionamento para ser possível fazer inferências sobre os resultados. As hipóteses nulas para cada ator pesquisado seguiram o padrão: "Não há correlação entre a importância atribuída ao relacionamento e o desenvolvimento de inovações nesse 
relacionamento". Assim, as hipóteses alternativas seguiram o modelo: "Há correlação entre a importância atribuída ao relacionamento e o desenvolvimento de inovações". Pelos testes estatísticos, foi verificado que a correlação entre as variáveis trabalhadas era bastante significativa porque os valores-p (Sig.) obtidos foram todos menores 0,05 , conforme demonstrações nos Quadros 1 a 7.

Quadro 1. Teste de Hipótese: correlação entre a relação com hotéis e o desenvolvimento de inovações

\begin{tabular}{|l|c|c|}
\hline $\begin{array}{l}\text { H0: Não há correlação entre a importância atribuída ao relacionamento com hotéis e o } \\
\text { desenvolvimento de inovações nesse relacionamento. }\end{array}$ & $\begin{array}{l}\text { Correlation } \\
\text { Coefficient }\end{array}$ &, 312 \\
\hline $\begin{array}{l}\text { H1: Há correlação entre a importância atribuída ao relacionamento com hotéis e o } \\
\text { desenvolvimento de inovações nesse relacionamento. }\end{array}$ & Sig. (2-tailed) &, 004 \\
\hline \multicolumn{2}{|c|}{ H0: rejeitada, há correlação } \\
\hline
\end{tabular}

Quadro 2. Teste de Hipótese: correlação entre a relação com agências de viagem e o desenvolvimento de inovações

\begin{tabular}{|l|c|c|}
\hline $\begin{array}{l}\text { H0: Não há correlação entre a importância atribuída ao relacionamento com agências de } \\
\text { viagem e o desenvolvimento de inovações nesse relacionamento. }\end{array}$ & $\begin{array}{l}\text { Correlation } \\
\text { Coefficient }\end{array}$ &, 352 \\
\hline $\begin{array}{l}\text { H1: Há correlação entre a importância atribuída ao relacionamento com agências de } \\
\text { viagem e o desenvolvimento de inovações nesse relacionamento. }\end{array}$ & Sig. (2-tailed) & ,001 \\
\hline \multicolumn{2}{|c|}{ H0: rejeitada, há correlação } \\
\hline
\end{tabular}

Quadro 3. Teste de Hipótese: correlação entre a relação com operadoras de turismo e o desenvolvimento de inovações

\begin{tabular}{|l|c|c|}
\hline $\begin{array}{l}\text { H0: Não há correlação entre a importância atribuída ao relacionamento com operadoras de } \\
\text { turismo e o desenvolvimento de inovações nesse relacionamento. }\end{array}$ & $\begin{array}{l}\text { Correlation } \\
\text { Coefficient }\end{array}$ &, 587 \\
\hline $\begin{array}{l}\text { H1: Há correlação entre a importância atribuída ao relacionamento com aoperadoras de } \\
\text { turismo e o desenvolvimento de inovações nesse relacionamento. }\end{array}$ & Sig. (2-tailed) &, 000 \\
\hline \multicolumn{2}{|c|}{ H0: rejeitada, há correlação } \\
\hline
\end{tabular}

Quadro 4. Teste de Hipótese: correlação entre a relação com instituição de ensino e o desenvolvimento de inovações

\begin{tabular}{|l|c|c|}
\hline $\begin{array}{l}\text { H0: Não há correlação entre a importância atribuída ao relacionamento com instituição de } \\
\text { ensino e o desenvolvimento de inovações nesse relacionamento. }\end{array}$ & $\begin{array}{l}\text { Correlation } \\
\text { Coefficient }\end{array}$ &, 244 \\
\hline $\begin{array}{l}\text { H1: Há correlação entre a importância atribuída ao relacionamento com instituição de } \\
\text { ensino e o desenvolvimento de inovações nesse relacionamento. }\end{array}$ & Sig. (2-tailed) &, 024 \\
\hline \multicolumn{2}{|c|}{ HO: rejeitada, há correlação } \\
\hline
\end{tabular}

Quadro 5. Teste de Hipótese: correlação entre a relação com associações de classe e o desenvolvimento de inovações

\begin{tabular}{|l|c|c|}
\hline $\begin{array}{l}\text { H0: Não há correlação entre a importância atribuída ao relacionamento com associações de } \\
\text { classe e o desenvolvimento de inovações nesse relacionamento. }\end{array}$ & $\begin{array}{l}\text { Correlation } \\
\text { Coefficient }\end{array}$ &, 224 \\
\hline $\begin{array}{l}\text { H1: Há correlação entre a importância atribuída ao relacionamento com associações de } \\
\text { classe e o desenvolvimento de inovações nesse relacionamento. }\end{array}$ & Sig. (2-tailed) &, 024 \\
\hline \multicolumn{2}{|c|}{ H0: rejeitada, há correlação } \\
\hline
\end{tabular}


Quadro 6. Teste de Hipótese: correlação entre a relação com organizações promotoras do destino e o desenvolvimento de inovações

\begin{tabular}{|l|c|c|}
\hline $\begin{array}{l}\text { H0: Não há correlação entre a importância atribuída ao relacionamento com organizações } \\
\text { promotoras e o desenvolvimento de inovações nesse relacionamento. }\end{array}$ & $\begin{array}{c}\text { Correlation } \\
\text { Coefficient }\end{array}$ & \multicolumn{1}{c|}{, 276} \\
\hline $\begin{array}{l}\text { H1: Há correlação entre a importância atribuída ao relacionamento com organizações } \\
\text { promotoras e o desenvolvimento de inovações nesse relacionamento. }\end{array}$ & Sig. (2-tailed) & ,010 \\
\hline \multicolumn{2}{|c|}{ H0: rejeitada, há correlação } \\
\hline
\end{tabular}

Quadro 7. Teste de Hipótese: correlação entre a relação com entidades governamentais e o desenvolvimento de inovações

\begin{tabular}{|l|c|c|}
\hline $\begin{array}{l}\text { H0: Não há correlação entre a importância atribuída ao relacionamento com entidades } \\
\text { governamentais e o desenvolvimento de inovações nesse relacionamento. }\end{array}$ & $\begin{array}{l}\text { Correlation } \\
\text { Coefficient }\end{array}$ & ,442 \\
\hline $\begin{array}{l}\text { H1: Há correlação entre a importância atribuída ao relacionamento com entidades } \\
\text { governamentais e o desenvolvimento de inovações nesse relacionamento. }\end{array}$ & Sig. (2-tailed) &, 000 \\
\hline \multicolumn{2}{|c|}{ H0: rejeitada, há correlação } & \\
\hline
\end{tabular}

Embora nenhum dos coeficientes de correlação aferidos tenha apresentado resultado acima de 0,6, pode-se dizer que, nesta pesquisa, as associações mais fortes entre as variáveis se deram nos casos dos relacionamentos com operadoras turísticas, com coeficiente de correlação 0,577, e com entidades governamentais, com coeficiente de correlação 0,442, conforme apresentado nos Quadros 3 e 7, respectivamente. Em seguida, com coeficientes de correlações menos altos ficaram os hotéis $(0,312)$ e as agências de viagem $(0,352)$, indicados nos Quadros 1 e 2. Finalmente, demonstrando uma correlação mais baixa entre as variáveis apareceram instituições de ensino $(0,244)$, associações de classe $(0,284)$ e organizações promotoras do destino $(0,276)$, como pode ser observado nos Quadros 4, 5 e 6.

\section{DISCUSSÃO DOS RESULTADOS}

Os resultados obtidos na pesquisa evidenciaram que a percepção dos gestores entrevistados convergia, em grande parte, com as descobertas apresentadas na recente literatura sobre inovação em turismo.

O fato de a grande parte dos entrevistados ter concordado que os seus relacionamentos eram importantes para o desenvolvimento de inovações e que a maioria destes era percebida como colaborativo convergiu com a literatura que enfatiza a importância dos relacionamentos de colaboração para a transferência de conhecimentos e recursos necessários à inovação no turismo. Pavlovich (2003), por exemplo, coloca que o estabelecimento de redes de relacionamentos é um fator crítico para o desenvolvimento de um destino turístico, pois contribuem para trocas intensivas de informação que permitem a criação de conhecimento compartilhado entre os parceiros, sendo determinante para a geração de inovações.

A respeito dos atores com os quais os gestores entrevistados reportaram ter relacionamentos, surpreendeu o fato das organizações governamentais terem sido mencionadas 
como relações pouco frequentes, dado que são identificadas na literatura como parceiros poderosos na formulação de políticas e na coprodução de instalações turísticas nos destinos. No entanto, mesmo esses relacionamentos sendo pouco frequentes, foram os que apresentaram uma das correlações mais fortes entre a percepção da importância do relacionamento e o desenvolvimento de inovações, sugerindo que a frequência dos contados não é um bom indicador para aferir a eficácia dos relacionamentos. A análise conjunta desses resultados revelou que as organizações turísticas pesquisadas são assertivas em relação aos seus relacionamentos com as organizações governamentais, especialmente no que concerne ao contexto atual em o destino está sendo foco de grandes investimentos em função dos megaeventos planejados para os próximos anos. Eventos como estes alavancam a demanda turística, que de acordo com Hjalager (2010) é um dos principais fatores para estimular as organizações no turismo a inovar. Por isso, há grandes oportunidades neste momento para estas empresas aproveitarem ao máximo esses relacionamentos para realizar os seus potenciais inovadores.

Ainda em relação à importância da demanda para o desenvolvimento de inovações, é pertinente notar que de acordo com dados do Ministério do Turismo Brasileiro (MTUR, 2011), desde que o Rio de janeiro foi anunciado como sede de megaeventos nos próximos anos, o destino tem se beneficiado de um número crescente de turistas. É possível a publicidade decorrente do anúncio destes grandes eventos esteja estimulando o desenvolvimento de inovações pelas empresas turísticas no destino pesquisado de forma a captar e suprir a demanda crescente por produtos turísticos de alto valor agregado. Essa inferência está baseada no fato de que os resultados da pesquisa indicaram, em diferentes escalas, que todas as organizações pesquisadas tinham desenvolvido algum tipo de inovação com seus parceiros. Aqui cabe destacar que os relacionamentos com as operadoras de turismo revelaram ser os mais relevantes para o desenvolvimento de inovações, apresentando a correlação mais forte encontrada nesta pesquisa. As principais inovações desenvolvidas com esses parceiros foram relatadas em produtos, serviços e logística. Esses dados são coerentes com o momento atual de crescimento da demanda do destino uma vez que são as operadoras que elaboram os roteiros e pacotes turísticos, buscando os melhores atrativos para os potenciais clientes. Para isso, elas precisam estabelecer parcerias diversas especialmente com hotéis e agências de viagens que fazem a intermediação direta com os turistas.

Entretanto, cabe ressaltar que as empresas turísticas pesquisadas precisam fazer com que as inovações desenvolvidas sejam capazes de suportar preços competitivos globalmente. Por exemplo, o processo de precificação de hotéis que estava sendo praticado para a Rio+20, no qual foi necessário intervenção governamental para uma negociação que evitasse o cancelamento de delegações internacionais, que estavam alegando preços abusivos (Mendes, 2012), é um fato negativo que pode frear o crescimento da demanda e, logo, reduzir a realização do potencial inovador do destino. Ademais, extrapolar o nível global de preços praticados no turismo é um risco para as empresas do destino pesquisado a médio e longo prazo, especialmente após os grandes eventos, pois pode afugentar a demanda internacional acarretando perdas para todos os 
envolvidos. Nesse sentido, é urgente investir em políticas de preços adequadas ao cenário global a fim de evitar a queda da demanda que pode trazer grandes limitações para o desenvolvimento de inovações futuras.

A baixa frequência dos contatos nos relacionamentos com institutos de ensino e pesquisa, assim como a baixa correlação que esses relacionamentos apresentaram no que tange ao desenvolvimento de inovações, por sua vez, convergiram com pesquisas prévias que mostraram os relacionamentos de colaboração com universidades como não sendo tão relevantes para o desenvolvimento de inovações no caso da indústria do turismo como na indústria de manufaturados. No entanto, no contexto de um destino localizado em um país em desenvolvimento, como é o caso do Rio de Janeiro, fortalecer relacionamentos com centros de estudo e pesquisa pode acelerar o processo de inovação ao fornecer capital humano qualificado para os empreendimentos e, ao mesmo tempo, contribuir para a inclusão social por meio do trabalho. Por isso, os gestores desta região poderiam ser beneficiados se dessem prioridade maior a esse tipo de parceria.

Com relação ao principal recurso compartilhado pelos gestores com seus parceiros capital informacional - os resultados da pesquisa também convergiram com a literatura. Estes resultados são particularmente importantes, pois, conforme evidenciaram Sundbo et al. (2007) e Pavlovich (2003), as trocas intensivas de informação permitem a criação de conhecimento compartilhado e a complementação de competências, fatores críticos ao desenvolvimento de inovação. Como visto no caso das organizações pesquisadas, os recursos compartilhados proporcionaram, principalmente, inovações em produtos e serviços, nos modelos de gestão adotados e nas normas institucionais do destino, como marcos regulatório e de investimento. Sobre este último, pode-se citar como exemplo o programa Pró Copa, do Banco Nacional de Desenvolvimento Econômico e Social (BNDES, 2012), com condições especiais de financiamento definidas em comum acordo com as partes envolvidas para aqueles que cumprirem metas de sustentabilidade em novos empreendimentos.

O fato de os resultados desta pesquisa terem evidenciado que as organizações atuantes no turismo do Rio de Janeiro tinham desenvolvido inovações por meio de suas redes de relacionamento e que estes relacionamentos colaborativos eram vistos como fundamentais para esse processo, no entanto, não esgota a temática nem determina que as inovações estejam em um nível adequado de desenvolvimento. Para isso, pesquisas futuras como as sugeridas na próxima seção deste artigo são necessárias.

\section{CONSIDERAÇÕES FINAIS}

Lembra-se que este artigo apresentou os resultados de uma pesquisa que buscou analisar a existência de relação significativa entre colaboração e inovação nas empresas que atuam no turismo, precisamente na cidade do Rio de Janeiro. Para isso, um estudo empírico com mais de noventa organizações atuantes em diferentes áreas do turismo foi empreendido. A análise dos 
resultados evidenciou que, na percepção dos gestores entrevistados, a maioria dos relacionamentos colaborativos com outras organizações era importante e proporcionava na prática o desenvolvimento de inovações, corroborando com a literatura sobre o tema.

No entanto, se verificou que as correlações mais fortes foram com as operadoras turísticas e com as entidades governamentais. A partir disso, foi possível inferir que esses são os principais parceiros para o desenvolvimento de inovação dentre as empresas que atuam no turismo da cidade do Rio de Janeiro.

Por outro lado, os resultados sugeriram que os relacionamentos com instituições de ensino e pesquisa eram fracamente correlacionados com o desenvolvimento de inovações, mais uma vez confirmando a teoria. Todavia, esses relacionamentos foram considerados importantes provedores de capital social e informacional para as organizações pesquisadas, o que pode indiretamente estar contribuindo com o processo de inovação das empresas turísticas. No entanto, a pesquisa mostrou que essas parcerias podem ser mais bem aproveitadas no contexto do destino enfocado. Uma das razões para atribuir mais relevância a esses parceiros pode ser justificada pela necessidade de capital humano. É notória a carência de mão de obra qualificada em diversos setores produtivos do país, inclusive no turismo. Nesse quesito, não só para a sobrevivência cotidiana das operações, mas também para o desenvolvimento de inovações, as universidades e centros de pesquisa são celeiros importantes para a atração desse capital produtivo. Além disso, para um destino que se encontra num país em desenvolvimento, o turismo tem grande potencial de inclusão social e as universidades podem ser parceiros muito relevantes nesse processo.

Adicionalmente, algumas outras considerações sobre esta pesquisa podem ser tecidas. Primeiramente, uma definição ampla de inovação foi oferecida aos gestores e a amostra foi composta de grandes organizações turísticas, com melhores condições de investir em inovação. Esses dados podem explicar o fato de que a maioria dos entrevistados relatou um alto percentual de concordância em relação ao desenvolvimento de inovações. Contudo, a análise das correlações mostrou que, apesar de haver correlação em todos os casos, não há uma relação tão forte entre a percepção de importância dos relacionamentos e a prática de inovação. No entanto, esses resultados não surpreendem, uma vez que a teoria, como vista neste artigo, considera a indústria do turismo moderadamente inovadora.

Dentre as possíveis lições e recomendações que podem ser elaboradas a partir dos resultados dessa pesquisa, destacam-se a criação de políticas públicas de financiamento para instituições de ensino e pesquisa em turismo para encorajar e fortalecer parcerias com essas organizações de forma a gerar novos conhecimentos e, assim, acelerar o processo de inovação em produtos e serviços de alto valor agregado. Além disso, como lição para os gestores das empresas turísticas reforça-se a necessidade de se estreitar os relacionamentos de colaboração para a criação de vantagem competitiva no longo prazo com a adoção de estratégias de diferenciação por meio de inovações tanto para a captação de demanda quanto para a fidelização dos clientes atuais. 
Em suma, a pesquisa em questão neste artigo contribuiu para a teoria ao corroborar a tese de que a colaboração é fundamental para o desenvolvimento de inovações na indústria do turismo. Além disso, mostrou que as maiores organizações que atuam no turismo do destino pesquisado, ao estabelecerem parcerias que de fato contribuem para a geração de inovações, estão investindo em competitividade no longo prazo, o que pode trazer vantagens para elas, especialmente, após o período dos megaeventos que o destino irá sediar.

Entretanto, o conhecimento aqui compartilhado deve ser enriquecido por novas pesquisas. Entrevistas em profundidade podem revelar mais detalhes sobre as inovações desenvolvidas nos relacionamentos colaborativos evidenciados e iluminar melhores práticas para o aprendizado de outras organizações no setor. Além disso, um levantamento dos principais desafios enfrentados pelas empresas no processo de inovação pode auxiliar na formulação de políticas públicas com vistas ao fomento do turismo no destino. A ampliação da amostra pode ainda permitir comparações entre organizações turísticas de diferentes tamanhos para um melhor entendimento dos comportamentos inovadores. A análise das características estruturais da rede pesquisada pode revelar novos insights para a gestão estratégica do destino. Enfim, o tema da inovação no turismo é prolífero para novas investigações uma vez que a teoria ainda está em processo de consolidação e há poucas pesquisas empíricas sobre o tema que adotam os destinos brasileiros como unidade de análise.

\section{REFERÊNCIAS}

BNDES. (2012). BNDES Automático - Condições especiais para o setor de hotelaria. Recuperado de http://www.bndes.gov.br/SiteBNDES/bndes/bndes_pt/Institucional/Apoio_Financeiro/Produtos/BNDES_A utomatico/condicoes_hotelaria.html.

Buhalis, D. (1999). Information technology for small and medium-sized tourism enterprises: adaption and benefits. Journal of Information Technology \& Tourism, 2(2), 79-95.

Donaire, D., Silva, M. P., \& Gaspar, M. A. (2009). A Rede de Negócios do Turismo: um estudo sobre duas características e implicações estratégicas. Turismo Visão e Ação, 11(1).

Gil, A. C. (2009). Métodos e Técnicas de Pesquisa Social. (6a ed.). São Paulo: Atlas.

Hair, J. F., Anderson, R. E., Tatham, R. L., \& Black, W. C. (2006). Análise Multivariada de Dados. (5a ed.). Porto Alegre: Bookman.

Hall, C. M. (2009). Innovation and tourism policy in Australia and New Zealand: never the twain shall meet? Journal of Policy Research in Tourism, Leisure and Events, 1(1), 2-18.

Hjalager, A-M. (2010). A review of innovation research in tourism. Tourism Management, 31, 1-12.

Hjalager, A-M. (2007). Stages in the Economic Globalization of Tourism. Annals of Tourism Research, 34(2), 437-457.

Hjalager, A-M. (2002). Repairing innovation defectiveness in tourism. Tourism Management, 23, 465-474.

Hjalager, A-M. (1997). Innovation patterns in sustainable tourism: An analytical typology. Tourism

Management, 18(1), 35-41. 
Likert, R. A. (1975). Organização Humana. São Paulo: Atlas.

March, R., \& Wilkinson, I. (2009). Conceptual Tools for Evaluating Tourism Partnerships. Tourism Management, 30.

Matínez-Ros, E., \& Orfila-Sintes, F. (2009). Innovation activity in the hotel industry. Technovation, 29, 632641.

Mayer, M. (2009). Innovation as Success Factor in Tourism: Empirical Evidence from Western Austrian Cable-Car Companies. Erdkunde, 63(2), 123-139.

Mendes, P. (10 maio 2012). Governo faz 'apelo' para que hotéis reduzam preços para o Rio+20. G1. Recuperado de http://g1.globo.com/natureza/rio20/noticia/2012/05/governo-faz-apelo-para-que-hoteisreduzam-precos-para-o-rio20.html.

MTUR (Ministério do Turismo). (2011). Estudo da demanda turística internacional 2004-2010. Brasília. Recuperado de http://www.dadosefatos.turismo.gov.br/export/sites/default/dadosefatos/demanda_turistica/internacion al/download_internacional/Demanda_Turxstica_Internacional_-_Fichas_Sinteses_-_2004-2010.pdf.

Orfila-Sintes, F., Crespi-Cladera, R., \& Martinez-Ros, E. (2005). Innovation activity in the hotel industry: Evidence from Balearic Islands. Tourism Management, 26, 851-865.

Pavlovich, K. (2003). The evolution and transformation of a tourism destination network: the Waitomo Caves, New Zealand. Tourism Management, 24, 203-216.

Pikkemaat, B. (2008). Innovation in small and medium-sized tourism enterprises in Tyrol, Austria. Entrepreneurship and Innovation, 9(3), 187-197.

Pikkemaat, B., \& Peters, M. (2005). Towards the measurement of innovation - a pilot study in the small and medium sized hotel industry. Journal of Quality Assurance in Hospitality \& Tourism, 6(3/4), 89-112.

Pikkemaat, B., \& Weiermair, K. (2007) Innovation through Cooperation in Destinations: First Results of an Empirical Study in Austria. Anatoila: An international Journal of Tourism and Hospitality Research, 18(I), 6783.

Plaza, B., Galvez-Galvez, C., \& Gonzalez-Flores, A. (2011). Orchestrating innovation networks in e-tourism: A case study. African Journal of Business Management, 5(2), 464-480.

Rodgers, M. Networks, Firm Size and Innovation. (2004). Small Business Economics, 22, 141-153.

Schumpeter, J. A. (1934). The theory of economic development: An inquiry into profits, capital, credit, interest and the business cycle. Cambridge: Harvard University Press.

Scott, N., Baggio, R., \& Cooper, C. (2008). Destination Networks: Four Australian Cases. Annals of Tourism Research, 35(1), 169-188.

Stamboulis, Y., \& Skayannis, P. (2003). Innovation strategies and technology for experience-based tourism. Tourism Management, 24, 35-43.

Stevenson, W. J. (2001). Estatística aplicada à administração. São Paulo: Habra.

Sundbo, J., Orfila-Sintes, F., \& Sørensen, F. (2007). The innovative behavior of tourism firms - Comparative studies of Denmark and Spain. Research Policy, 36, 88-106.

Timur, S., \& Getz, D. (2008). A network perspective on managing stakeholders for sustainable urban tourism. International Journal of Contemporary Hospitality Management, 20(4), 445-461, 2008. 
Tremblay, P. (1998) The Economic Organization of Tourism. Annals of Tourism Research, 5(4), 837-859.

Victorino, L., Verma, R., Plaschka, F., \& Dev, C. (2005). Service innovation and customer choices in the hospitality industry. Managing Service Quality, 15(6), 555-576.

Wang, Y., \& Krakover, S. (2008). Destination Marketing: Competition, Cooperation or competition? International Journal of Contemporary Hospitality Management, 20(2), 126-141.

Artigo recebido em: 06/06/2012.

Artigo aprovado em: 07/11/2012. 